\title{
Zur Genese der Aareschlucht (Berner Oberland, Schweiz)
}

\section{Zusammenfassung}

Eine statistische Analyse der Kluft- und Talrichtungen im Gebiet der Aareschlucht (Berner Oberland, Schweiz) ergibt, daß diese Richtungen übereinstimmen und somit die Aareschlucht nicht durch epigenetische Flußerosion, d. h. durch ein Durchsägen des emporgestaucht werdenden Kirchetriegels in verschuppten Unterkreidekalken mit alttertiärer Brekzie und Sandsteinen entstanden ist, sondern durch tektonische Klüftung vorgezeichnet war. Dabei wurden die Kluftspalten höchstens ausgeräumt: die Kolkung am Schluchtfuß ist minimal. Es ist durchaus möglich, daß die Klüftung noch viel tiefer reicht und durch den Fluß nicht nur nicht erodiert, sondern bis zur vermeintlichen Erosionsbasis aufgefüllt wurde.

\begin{abstract}
On the genesis of the Aare Gorge, Bernese Oberland, Switzerland: A statistical analysis of the directions of joints and river segments in the region of the Aare Gorge yields that these directions coincide and that therefore the Gorge was not created by epigenetic river erosion in lower Cretaceous limestones with early Tertiary breccias and sandstones, but that it was predesigned by tectonic joints. In this instance, the joint-clefts were at most cleared out: the erosion at the foot of the gorge is minimal. It is quite possible that the preexisting joint-clefts reach quite deep down and were not only not "cut" by the Aare river, but filled by sediments up to the supposed base level of erosion.
\end{abstract}

\section{Einleitung}

In der Aareschlucht, einer der beliebtesten Touristenattraktionen des Berner Oberlandes, durchquert die Aare zwischen den Talböden von Innertkirchen und Meiringen einen Felsriegel, den Kirchetriegel, in einer engen, 1400 m langen Schlucht; durch sie führt ein 1889 kühn angelegter Touristenweg mit Galerien und Stegen. Hinsichtlich der Genese der Schlucht wird immer wieder behauptet, sie sei von der Aare in den emporgestauchten Kirchetriegel epigenetisch eingesägt worden. Die gegenwärtigen Autoren hegten ernste Zweifel an der Richtigkeit dieser Deutung; sie haben daher eine Untersuchung zur Genese der Aareschlucht vorgenommen. Dazu wurde die Hauptschlucht von oben (SE) nach unten (NW) auf dem Touristenweg begangen. Anschließend wurden in der Muer-Lammi bis zum Restaurant Lammi am Kopf der Finsteren Schlucht Kluftmessungen vorgenommen.
In der Aareschlucht wurden in Weitungen Schwemmebenen mit reichem Erratikergeschiebe festgestellt. Abb. 1 zeigt das Gebiet 1:20700 mit den eingetragenen Kluftmeßstellen. Die Messungen wurden ausgewertet und mit der Gestalt der Schlucht verglichen. Es konnte eindeutig festgestellt werden, daß Kluft- und Flußsegmentrichtungen miteinander übereinstimmen. Daraus geht hervor, daß die Schlucht durch die Klüftung vorgezeichnet war und nur Kluft- und Glazialschutt vom Fluß ausgeräumt wurden.

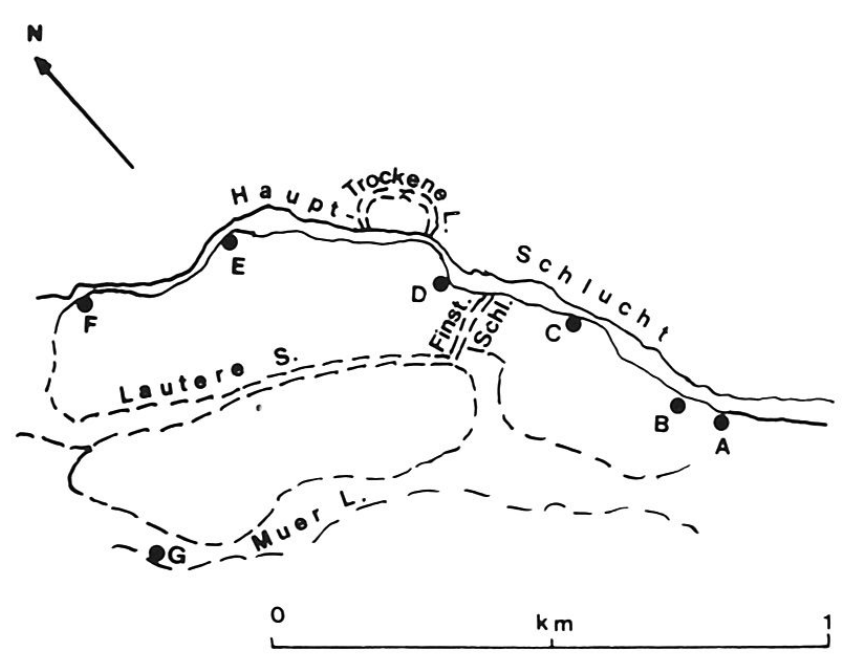

Abb.1 Plan der Umgebung der Aareschlucht.

\section{Geologie und Geomorphogenese}

Die Geologie und die Geomorphogenese des Aareschluchtbereiches wurden schon von ARBENZ und MÜLLER $(1924,1927,1934)$ und MÜLLER (1938) eingehend beschrieben.

Danach ist oberhalb von Meiringen das Tal der Aare scheinbar abgeschlossen durch den Felsriegel des «Kirchet», der sich 150-200 m über die Talsohle erhebt. Nach

R. Hantke, Prof. Dr., Geologisches Institut, ETH Zentrum, 8092 Zürich

A. E. Scheidegger, Prof. Dr., Abteilung Geophysik, TU Wien, A-1040 Wien 
der gängigen Lehrmeinung wäre der Riegel von der Aare «durchsägt» worden; früher außer in der jetzigen auch in älteren «epigenetischen» Schluchten: der Trockenen Lamm, der Lauteren Schlucht, der Muer-Lammi und der Finsteren Schlucht (MÜLLER, 1938) sowie in einer weiteren, von STAEGER bei geologischen Kartierungsarbeiten für Blatt 1210 Innertkirchen des Geologischen Atlas der Schweiz 1:25000 festgestellten verfüllten Schlucht (in HANTKE, 1991). Danach wäre die Aareschlucht noch jüngst «ein prachtvolles Beispiel für die Tiefenerosionskraft des Wassers" (LABHART, 1992, Abb. 74). Die Erosionskraft wirkt sich jedoch vor allem in Lockergesteinen aus, namentlich wenn sie von der schützenden Vegetationsdecke entblößt sind. Im Felsgestein ist sie um Zehnerpotenzen kleiner.

Nach MÜLLER (1938) liegen im Kirchetriegel Schichtkomplexe vor, die vorwiegend aus dichten Kalken bestehen. Diese bedecken als autochthone Gesteinsfolgen von oberstem Malm und unterster Kreide das Innertkirchner Kristallin des Aarmassivs, ein von Gneisschollen durchsetztes granitisches Gestein. Ihnen liegen - eine fast 100 Ma dauernde Festlandperiode belegend - erst wieder alttertiäre geringmächtige verschieferte Mürrenbrekzie, kohliger Kalk und priabone Sandkalke auf. Sie trennen höhere Unterkreideschuppen. Im engeren Gebiet der Aareschluchten sind 5 solcher Schichtkomplexe

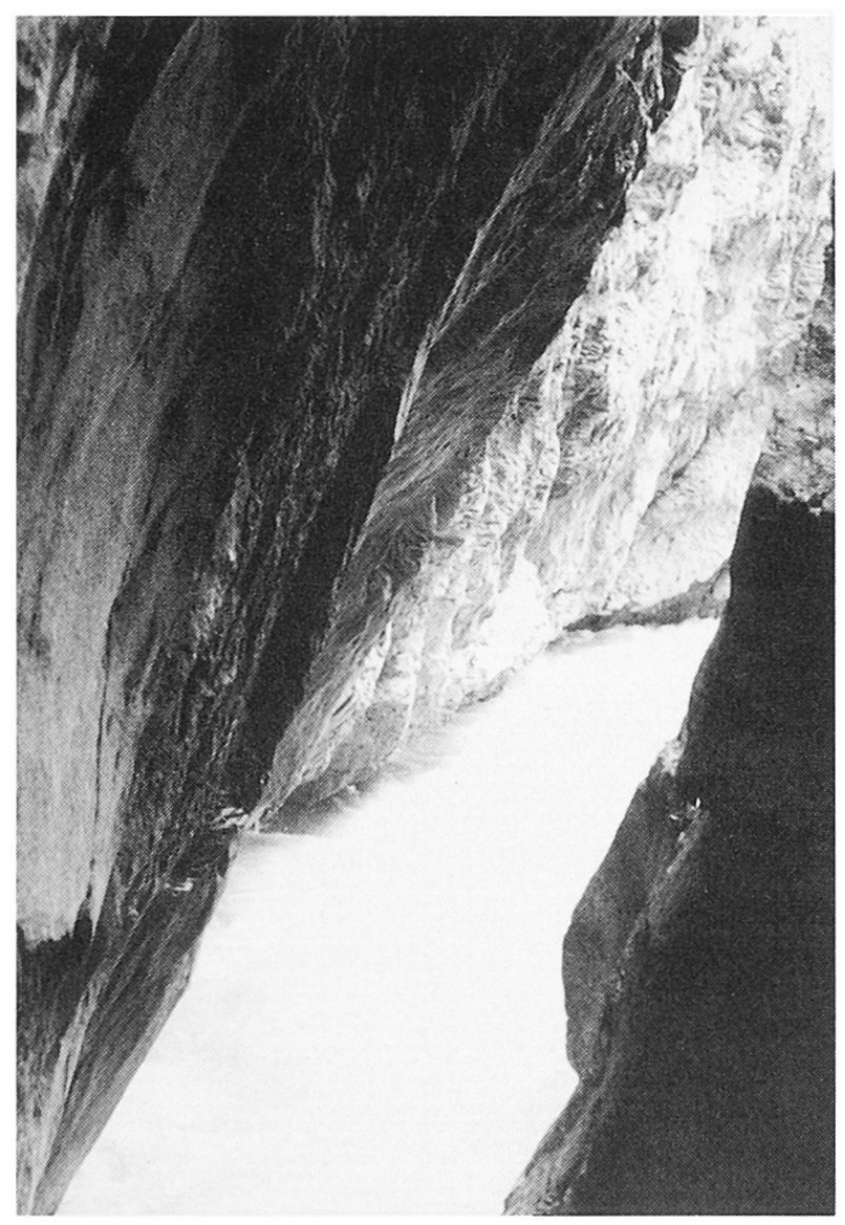

Abb.2 Schief stehende Engstelle der Aareschlucht.

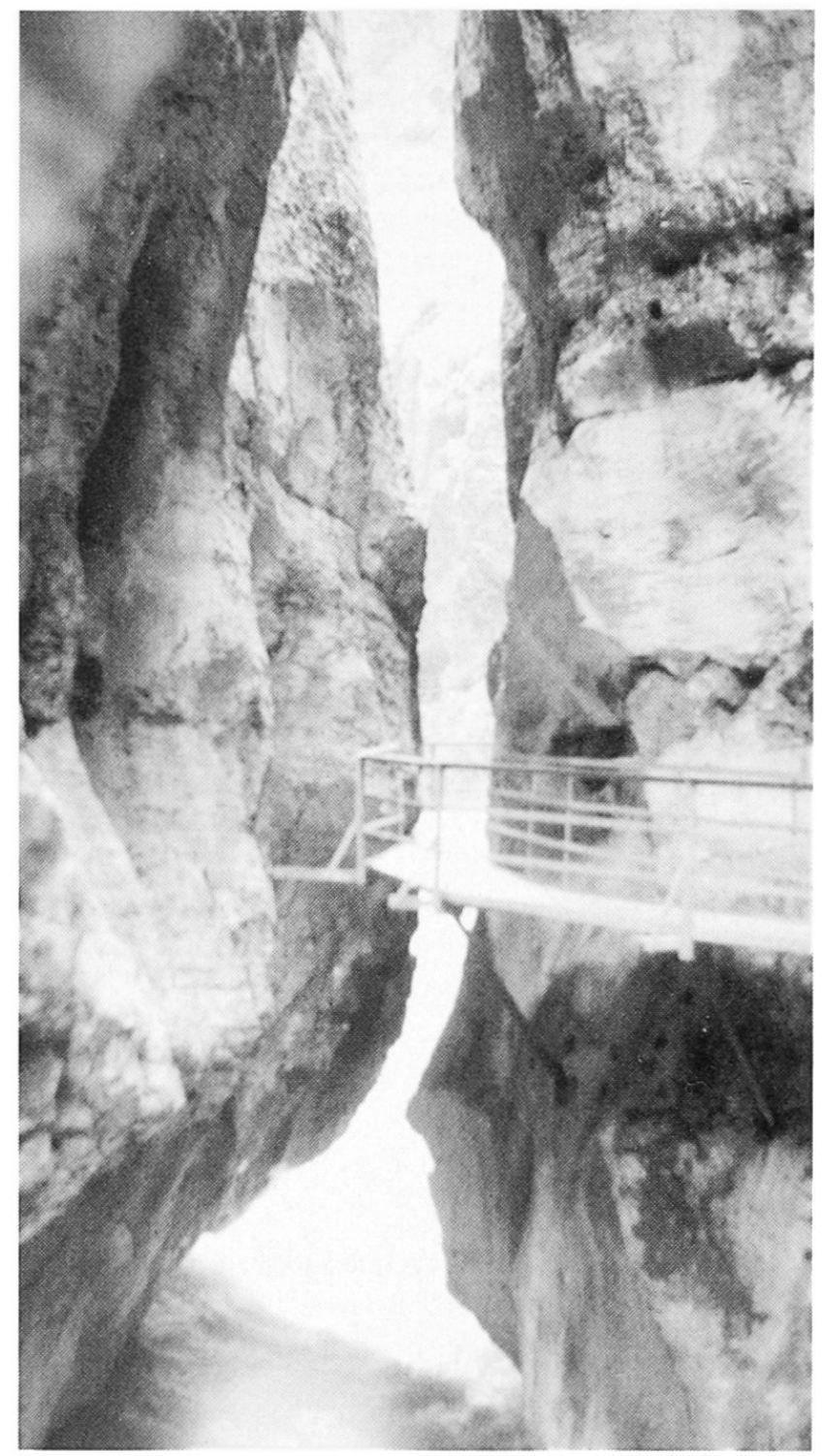

Abb. 3 Verbreiterung der Schlucht am Schluchtfuß; steile Klüftung.

übereinander festgestellt worden, die alle zur basalen Sedimentbedeckung des Aarmassivs gehören (MÜLLER, 1938). Längs schaufelförmigen (listrischen) Flächen wurde die autochthone Gesteinsserie bei der Überschiebung der Wildhorndecke verschert und verschuppt.

Die Sequenzen sind in der Aareschlucht von oft engen, z. T. nur meterbreiten Durchbrüchen durchsetzt, die mit etwas weiteren Stellen abwechseln. Die Schluchtwände stehen oft nicht vertikal, sondern steil schief (Abb. 2); noch weit schiefer stehen die Wände der Taminaschlucht hinter Bad Ragaz (Kt. St. Gallen). Dies läßt die These einer «Durchsägung» des kompakten Felsgesteins durch den Fluß schon rein morphologisch unwahrscheinlich erscheinen, da diese vertikal erfolgen müßte. Die Kolkung am Wandfuß ist gering; sie folgt kluftbedingt zickzackartig angeordneten Abschnitten. Zuweilen verbreitert sich der Spalt nach unten (Abb. 3). Die Finstere und 
die Lautere Schlucht sind einmündende Nebenschluchten. In ihnen liegen verkeilte Felsblöcke: bei der Klüftung niedergebrochenes Gesteinsgut und Erratiker. In den verschiedenen Kaltzeiten wurden die Schluchten jeweils durch Schuttmassen - vor allem Kluftschutt und Moränenmaterial - verbaut, so daß die Aare in den Spätphasen wieder neue Ausgänge aufzuspüren hatte. Sie fand solche in den verschiedenen Schluchten vor. Dabei wurden die einzelnen Schluchten von den Schmelzwässern der Aare aus dem jeweils von einem See gefüllten Innertkirchner Becken teilweise wieder etwas ausgeräumt. Diese hatte sie aber nicht einzutiefen, wohl aber zusammen mit der ebenfalls längs Klüften erfolgten Frostsprengung - durch Lösung da und dort zu erweitern vermocht. Dies dürfte vor allem durch Schmelzwässer aus dem zunächst noch von Eis gefüllten Becken von Innertkirchen geschehen sein. Die Wirkung der Lösung an Kalkstein ist recht bescheiden, wie HANTKE (1982) an Karstschemeln im Gebiet der Silberen (Kt. Schwyz) zeigen konnte. Da die Schlucht stellenweise nur $1 \mathrm{~m}$ breit ist, bewegt sich die Lösungswirkung maximal in dieser GröBenordnung, wahrscheinlich war sie deutlich geringer. Die in den Weitungen der Schlucht liegenden Erratiker von Aarekristallin wurden ausgeschmolzen und blieben liegen.

\section{Kluftsysteme}

Schon MÜLLER (1938) hat 4 Kluftsysteme unterschieden, alle nahezu $90^{\circ}$ einfallend. Die Streichrichtungen MÜLLERS wurden zum Vergleich mit den weiteren Ausführungen in unsere Notation umgerechnet; wir geben das Azimut der Fallrichtungen N-E und den Fallwinkel in Graden.

1. N38W streichend, unsere Notation $052 / 90$ oder 232/90

2. N69W streichend, unsere Notation 021/90 oder 201/90

3. N87W streichend, unsere Notation $003 / 90$ oder $183 / 90$

4. N30E streichend, unsere Notation 120/90 oder 300/90

Um die MÜLLERschen Daten zu verifizieren, haben wir selbst am oberen Ende (Locs. A, B., Tithonkalk, Abb. 4), in der Mitte (Locs. C, D, E, Öhrlikalk, Abb. 5) und am unteren Ende (Loc. F, Mürrenbrekzie), einschließlich der Muer-Lammi (Loc. G, siderolithischer Öhrlikalk), Klüfte gemessen. Es ergab sich kein signifikanter Unterschied der Kluftrichtungen an den verschiedenen Lokationen. Daher wurden die Klüfte gesamthaft nach der Methode von KOHLBECK und SCHEIDEGGER (1977) ausgewertet. Das entsprechende Poldichtediagramm wird in Abb. 6 wiedergegeben. Die statistische Auswertung nach KOHLBECK und SCHEIDEGGER (1977) erbrachte:

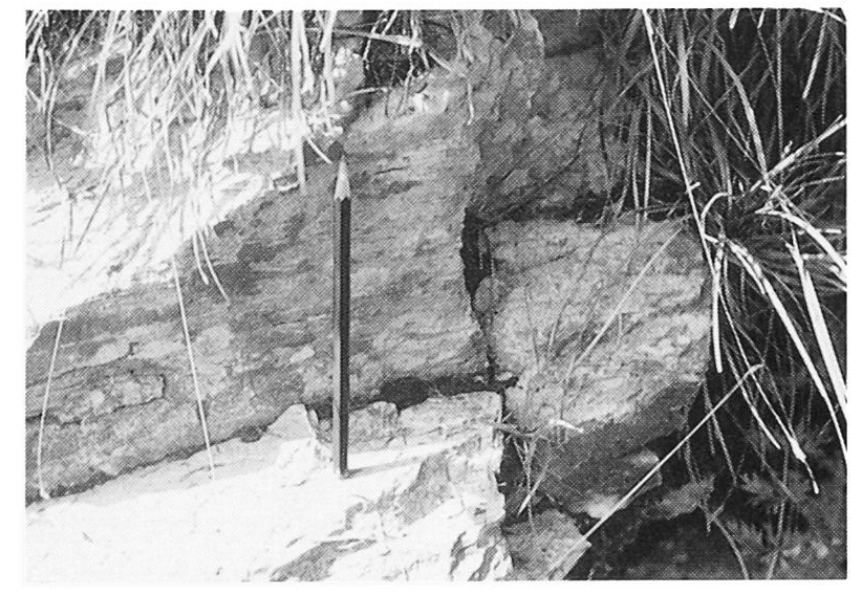

Abb. 4 Klüfte im Tithonkalk am Schluchteingang.

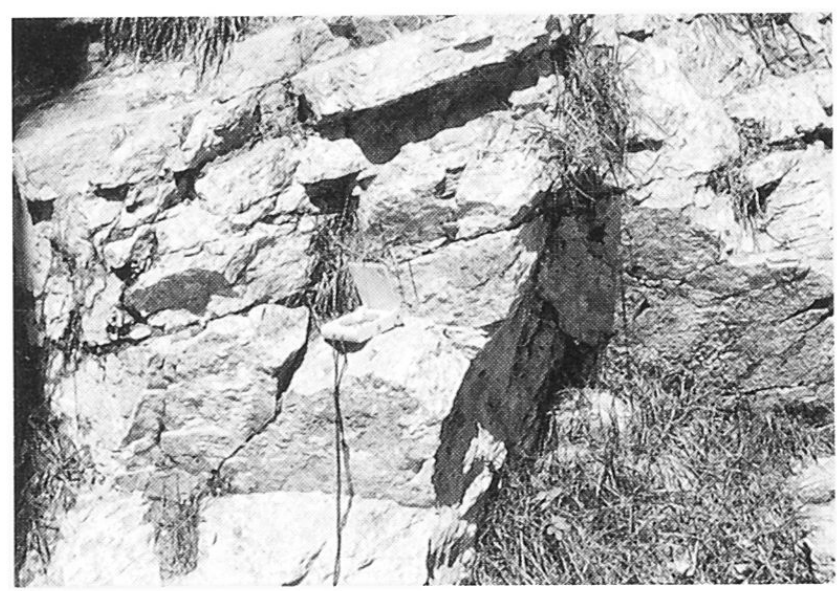

Abb. 5 Klüfte im Öhrlikalk in der Schluchtmitte.

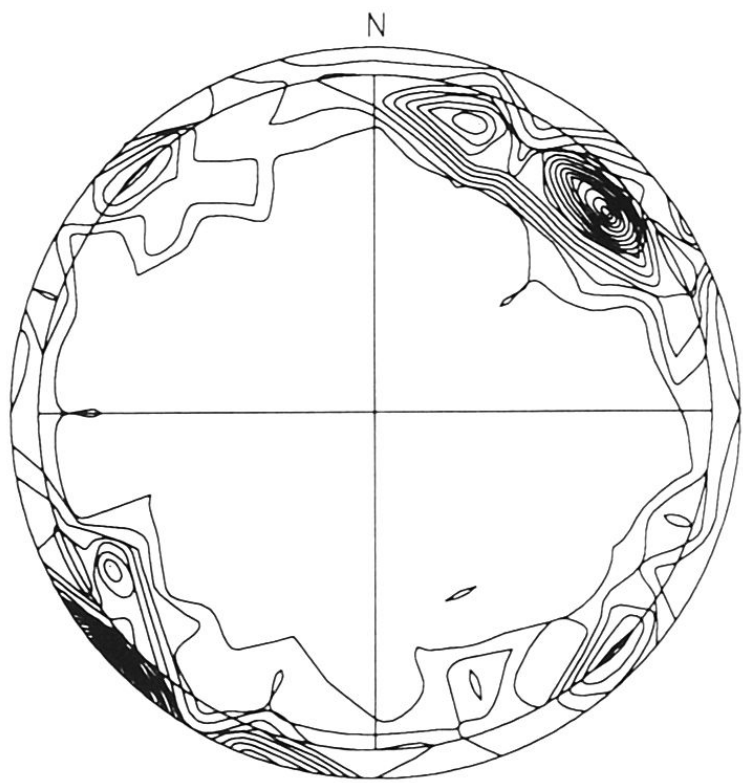

aareschlucht LAMBERT PROJECTION

Abb. 6 Poldichtediagramm der Klüfterichtungen. 
2 Kluftscharen:

Max. $1 \quad 223 \pm 1 / 84 \pm 8 \quad$ Gewicht 0,69

Max. $2 \quad 145 \pm 0 / 89 \pm 0 \quad$ Gewicht 0,31

Die von uns bestimmten zwei Kluftmaxima stimmen offensichtlich nur mäßig mit den von MÜLLER (1938) ermittelten Kluftscharen überein (Diskrepanz von $9^{\circ}$ zwischen unserem Maximum 1 und MÜLLERs Kluftschar 1; keine Entsprechung gibt es für unser Maximum 2). Die Auswertung nach KOHLBECK und SCHEIDEGGER (1977) wurde daher auch für 3 und 4 Scharen vorgenommen. Es ergaben sich folgende Resultate:

3 Kluftscharen:

Max. $1231 \pm 4 / 84 \pm 4$ Gewicht 0,50

Max. $2140 \pm 12 / 90 \pm 8$ Gewicht 0,28

Max. $3195 \pm 13 / 83 \pm 8$ Gewicht 0,22

4 Kluftscharen:

Max. $1231 \pm 7 / 84 \pm 5$ Gewicht 0,50

Max. $2139 \pm 11 / 90 \pm 10$ Gewicht 0,27

Max. $3194 \pm 11 / 84 \pm 8$ Gewicht 0,22

Max. $4336 \pm 29 / 50 \pm 22$ Gewicht 0,01

Die vierte Verteilung ist offensichtlich nicht signifikant, d. h., sie existiert nicht. Von den restlichen drei Verteilungen stimmt unser Maximum 1 (Diskrepanz $1^{\circ}$ ) mit der Kluftschar 1 von MÜLLER und unser Maximum 3 leidlich (Diskrepanz $6^{\circ}$ ) mit seiner Kluftschar 2 überein. Für die anderen von MULLLER angegebenen Kluftscharen gibt es bei uns keine Entsprechung. Da MÜLLER keine statistische Analyse gemacht, sondern offenbar nach Gefühl gearbeitet hat, muß die recht genaue Übereinstimmung von zwei unserer Maxima mit seinen zwei ersten (Haupt?-) Verteilungen als durchaus positiv gewertet werden. Nach unseren Messungen existieren somit nur drei signifikante Kluftscharen, die statistisch bestimimt wurden. Von diesen Kluftrichtungen ist übrigens die dritte mit einer der sonst in der Schweiz üblichen «europäischen" Kluftrichtungen (siehe SCHEIDEGGER, 1977) konform; die zwei anderen müssen lokal bedingt sein.
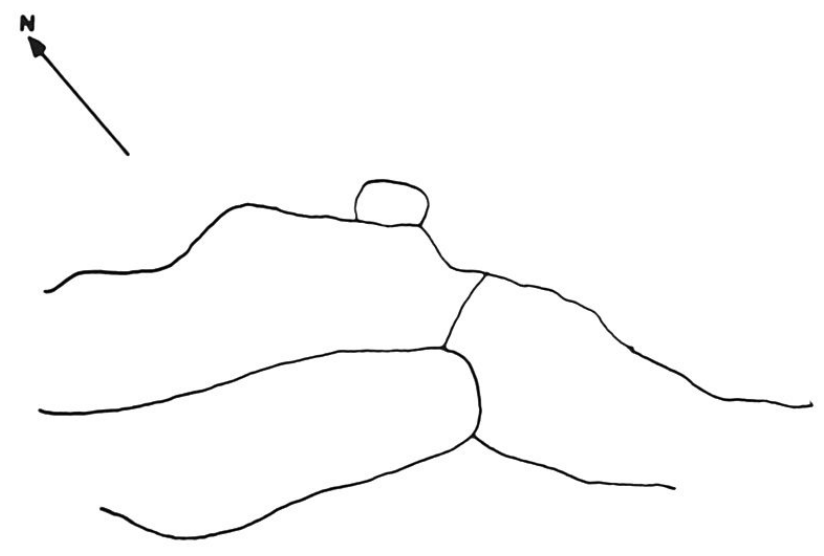

o $\quad \mathrm{km}$

Abb. 7 Mittellinien der Schluchten.

\section{Flußrichtungen}

Als nächstes galt es, Kluft- und Flußrichtungen miteinander zu vergleichen. Schon MÚLLER (1938) hat bemerkt, daß die "Aareschlucht hauptsächlich der N38W (in unserer Notation 52/90 oder 232/90) gerichteten Klüftung folgt".

Um dies zu verifizieren, wurden die Aareschlucht und die alten Nebenschluchten aus der Karte 1:10 000 herausgezeichnet und durch ihre Mittellinien ersetzt, jetzt ebenfalls 1:20700 (Abb. 7). Entlang der letzteren wurden Segmente von $5 \mathrm{~mm}$ ( $50 \mathrm{~m}$ in natura) Länge abgetragen und deren Richtung bestimmt.

Beim Hauptfluß wurden die resultierenden 31 50-m-Segmente bei einer totalen Länge von 1,55 km analysiert. Die Polrose der Verteilung der Segmente ist in Abb. 8 dargestellt. Nach KOHLBECK und SCHEIDEGGER (1977) ergab sich:

Eine Verteilung:

Maximum 51 oder $231 \pm 8$

Zwei Verteilungen:

Max. $1 \quad 09$ oder $189 \pm 10 \quad$ Gewicht 0,19

Max. 261 oder $241 \pm 0$ Gewicht 0,81

Drei Verteilungen:

Max. 158 oder $238 \pm 0$ Gewicht 0,82

Max. 210 oder $190 \pm 0$ Gewicht 0,16

Max. 3146 oder $326 \pm 0$ Gewicht 0,02

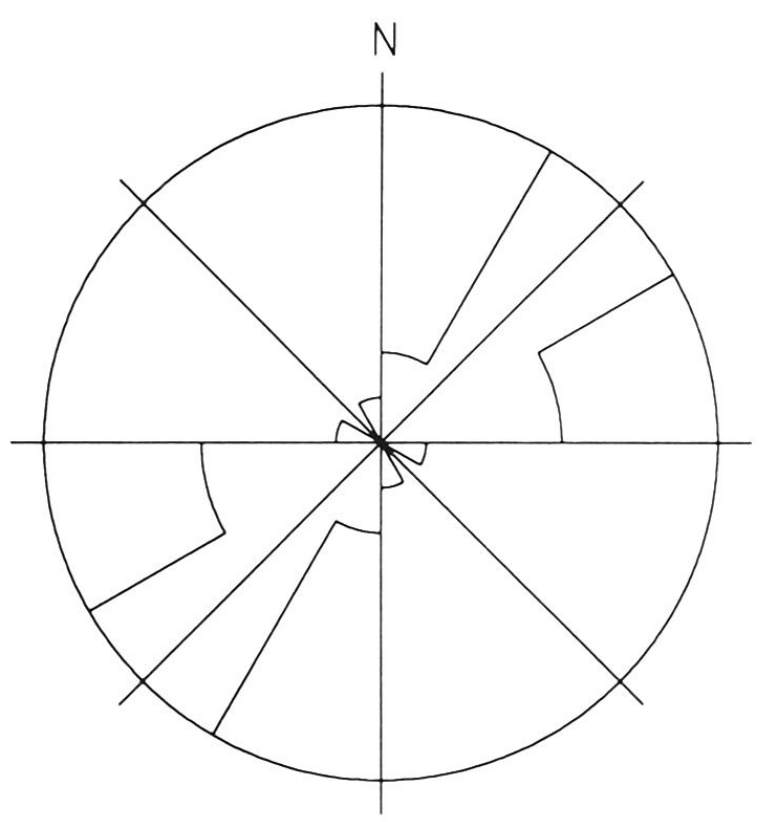

Aareschlucht Hauptfluss

INTERVALL $30.0 \quad$ MAXIMUM $=49 \%$ 
Ein Vergleich mit den Kluftmaxima zeigt, daß das Hauptflußsegmentmaximum (bei 2 oder 3 Verteilungen) jeweils gut dem Hauptmaximum der Klüfte entspricht. Dabei ist die Entsprechung nur einer Verteilung bei den Flußsegmenten am genauesten: Der Fluß folgt im Mittel der Hauptkluftschar. Aber auch die Nebenmaxima bei 3 Verteilungen stimmen recht gut mit den Nebenmaxima der Klüfte bei 3 Kluftverteilungen überein. Es zeigt sich somit, daß die Flußrichtungen durch die Kluftrichtungen vorgezeichnet sind.

Die Prozedur wurde unter Einbeziehung der Nebenschluchten wiederholt. Es resultierten nun 82 50-m-Segmente. Die Polrose ist in Abb. 9 wiedergegeben. Die statistische Auswertung ergab folgende Resultate:

Eine Verteilung:

Maximum 41 oder $221 \pm 3$

Zwei Verteilungen:

Max. 143 oder $223 \pm 7$ Gewicht 0,83

Max. 2144 oder $324 \pm 21$ Gewicht 0,17

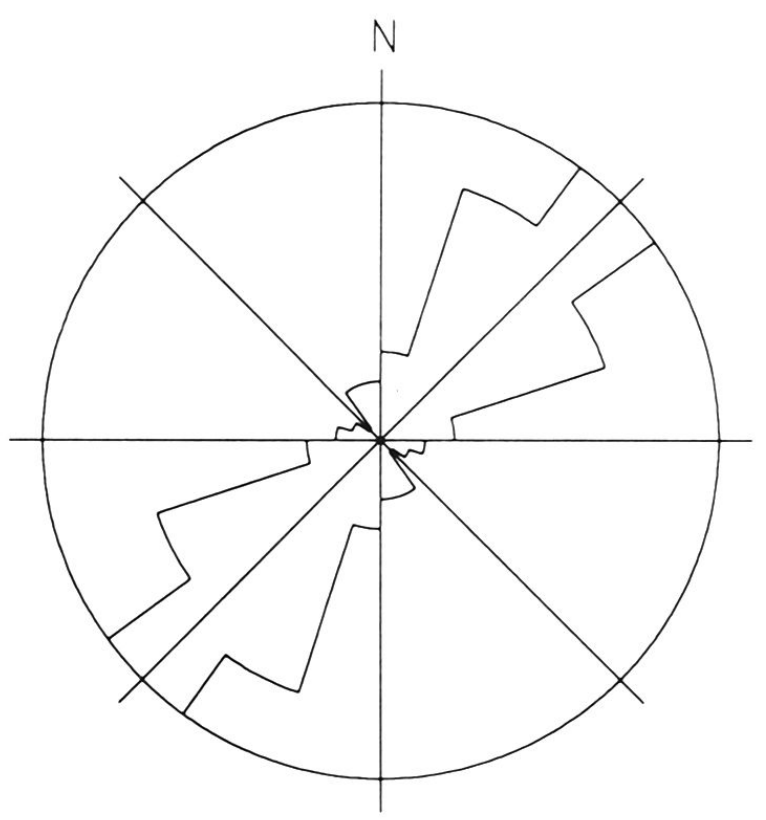

Aare- und Nebenschluchten

INTERVALL 18.0 MAXIMUM= $29 \%$

Abb. 9 Polrose der Segmentrichtungen der Aareschlucht zuzüglich der umgebenden Schluchten.
Drei Verteilungen:

Max. 153 oder $233 \pm 1$ Gewicht 0,55

Max. 2143 oder $323 \pm 26$ Gewicht 0,17

Max. 322 oder $202 \pm 0$ Gewicht 0,28

Das Bild bleibt somit, abgesehen von den etwas größeren Diskrepanzen, dasselbe wie bei der Hauptflußrichtung der Aare ohne Nebenschluchten.

\section{Schlußfolgerung}

In Anbetracht der Übereinstimmungen zwischen Kluftund Flußrichtungen ergibt sich, daß diese durch die Klüfte vorgezeichnet waren und daß die Aare den Kirchetriegel nicht "epigenetisch durchsägt" hat, sondern ganz einfach bestehenden Kluftsystemen gefolgt ist. Dabei wurde nur wenig seitlich ausgekolkt, und zwar hauptsächlich bis auf das die Talböden von Innertkirchen und Meiringen verbindende Erosionsniveau, wie die Erweiterung der Rinne an der Basis (Abb. 3) zeigt. Es ist zu erwarten, daß die Kluftspalten sogar tiefer, etwa bis auf das Niveau der Aalenienschiefer, reichen und bis auf das Erosionsniveau der Talböden mit Sedimenten angefüllt wurden. Diese Vermutung ist allerdings durch weitere Untersuchungen zu prüfen.

\section{Literatur}

ARBENZ, P., MÜLLER, F. (1924): Das Profil des Kirchetriegels bei Meiringen. In: Eclogae geol. Helv. 20(2): 236-241.

ARBENZ, P., MÜLLER, F. (1934): Exkursion Nr. 49, Die Aareschlucht des Kirchet (Meiringen-Innertkirchen). In: Geol. Führer Schweiz 9: 668-671.

HANTKE, R. (1982): Zur Talgeschichte des Gebietes zwischen Pragel- und Klausenpaß. In: BETTSCHART, A., et al. (Hg.), Die Karstlandschaft des Muotatales, Berichte Schwyz. Naturforsch. Ges. 8: 3-12.

HANTKE, R. (1991): Landschaftsgeschichte der Schweiz und ihrer Nachbargebiete, Thun, $312 \mathrm{~S}$

KOHLBECK, F., SCHEIDEGGER, A. E. (1977): On the theory of the evaluation of joint orientation measurements. In: Rock Mechanics 9: 9-25.

LABHART, T. P. (1992): Geologie der Schweiz, Thun, $211 \mathrm{~S}$. MÜLLER, F. (1938): Das Gebiet der Aareschlucht. Geologie der Engelhörner, der Aareschlucht und der Kalkkeile bei Innertkirchen, Berner Oberland (S. 42-47). In: Beitr. Geol. Karte Schweiz, N. F. 74: I-X, 1-55.

SCHEIDEGGER, A. E. (1977): Kluftmessungen im Gelände und ihre Bedeutung für die Bestimmung des tektonischen Spannungsfeldes in der Schweiz. In: Geographica Helvetica 32: 121-134. 